\title{
Array signal processing and systems
}

\author{
Bin Liao' ${ }^{1}$ - Arjuna Madanayake ${ }^{2}$. \\ Panajotis Agathoklis ${ }^{3}$
}

Published online: 15 February 2018

(C) Springer Science+Business Media, LLC, part of Springer Nature 2018

We are delighted to present this special issue of Multidimensional Systems and Signal Processing on Array Signal Processing and Systems. Sensor arrays play an important role in spatio-temporal signal processing with applications spanning across multiple fields such as electromagnetic, acoustics, ultrasonic and seismic processing systems. This plethora of possible applications has sparked a large number of new theoretical developments and array processing systems in the last few years.

In the field of electromagnetic sensor (antenna) arrays, there is much interest in the electronically-steerable radio-frequency $(\mathrm{RF})$ beams that can be obtained from phased-array antennas. In the microwave bands, phased-array antennas take both analog and digital signal processing approaches, and find applications in wireless base-stations, radar sensors, space communications, and radio telescopes. In the emerging mm-wave regime, modern applications of array processing are of paramount importance for wireless communications. In particular, emerging 5G systems based on massive-MIMO basestations depend on array processing to mitigate the effects of high path loss and blockages in an urban environment. Acoustic sensor (microphone) arrays are extremely useful for audio and multimedia applications, including high-fidelity sound recording, immersive multimedia and augmented/virtual reality. Ultrasound sensor arrays find extensive applications in biomedical engineering where they are used for imaging the human body and in structural health monitoring of airframes

Bin Liao

binliao@szu.edu.cn

Arjuna Madanayake arjuna@uakron.edu

Panajotis Agathoklis panagath@ece.uvic.ca

1 College of Information Engineering, Shenzhen University, Shenzhen 518060, China

2 Department of Electrical and Computer Engineering, The University of Akron, Akron, OH, USA

3 Department of Electrical and Computer Engineering, University of Victoria, Victoria, Canada 
and other structures in harsh environments. This Special Issue aims to present recent important developments of array signal processing and systems. Both original research articles and review articles in relevant fields are covered.

Following an open call for papers, seventeen articles are included in this Special Issue. More precisely, the Special Issue includes one review article and sixteen research articles. In the review article by Romanofsky and Toonen (2016), a brief history of developments which led to the realization of array antennas based on ferroelectric thin films is presented and key performance differences provided by competing thin film deposition techniques are highlighted. Moreover, the authors discuss the outlook of the impact that voltage-controlled magnetism and magnetoelasticity (provided by emerging multiferroic thin films) will have on future array antenna technologies.

The remaining 16 articles can be generally grouped into three thematic areas: (1) parameter estimation using sensor arrays; (2) beamforming for sensor arrays, and (3) signal processing in phased-array and multiple-input multiple-output (MIMO) radars as detailed below.

\section{Parameter estimation using sensor arrays}

In this group, we have 9 papers, which mainly focus on the estimation of direction-ofarrival (DOA) and frequency. The paper by Liu et al. (2016) presented an algorithm for two-dimensional DOA estimation of noncircular sources using an L-shaped sparse array composed of two co-prime arrays. In this method, the array aperture can be significantly increased, while the computational complexity is still acceptable since the azimuth angles can be estimated without peak searching and eigenvalue decomposition. Simulation results show that this method can provide improved performance in terms of the estimation accuracy and resolution.

Ali Khan et al. (2016) proposed a novel DOA estimation algorithm that uses the adaptive directional $t-f$ distribution (ADTFD) for the analysis of close signal components. This algorithm optimizes the direction of kernel at each point in the $t-f$ domain to obtain a clear $\mathrm{t}-\mathrm{f}$ representation, which is then exploited for DOA estimation. Experimental results indicate the use of adaptive directional TFD outperforms other TFDs in terms of resolution and cross-term suppression properties. This method also gives good results for sparse signals.

In the paper by Zhang et al. (2017), a new method is presented to effectively estimate the signal DOA and the phase error of a uniform linear array. Assuming that one sensor has been calibrated, this method appropriately reconstructs the data matrix and establishes a series of linear equations with respect to the unknown parameters through eigenvalue decomposition. The unknown parameters can be determined directly by the least squares method. Unlike the conventional methods, the proposed method only requires one calibrated sensor, which may not be consecutively spaced to the reference one. The computational complexity analysis is given and the effectiveness of the proposed method is validated by simulation results.

In the paper by Li et al. (2016a), a new DOA estimation method is proposed using a rotational uniform linear array (RULA) consisting of omnidirectional sensors. The main contribution of this method is that the number of distinguishable signals is larger than the methods in the literature with a uniform linear array consisting of the same number of omnidirectional sensors. Moreover, the new method can effectively reduce unknown spatial noises using a generalized complement projection matrix under the RULA framework. Extensive simulations demonstrate that the performance of RULA is satisfactory under various circumstances. 
The paper by Astapov et al. (2017) discussed the problem of distinguishing gunshot Shockwave (SW) and Muzzle Blast (MB) gunshot events in a scenario with presence of NOI acoustic events, where the MB transient is not guaranteed to strictly follow the SW transient. A shooter localization procedure comprising gunshot acoustic event identification based on DOA information, gunshot geometry estimation and shooter position estimation was presented and verified on real-life data. The main advantages of the proposed localization procedure include its ability to operate asynchronously in a size-invariant WSN, low dependency on gunshot parameter assumptions and increased noise tolerance.

The paper by $\mathrm{Wu}$ et al. (2016) conducted an explicit theoretical analysis of the characteristics of the phase difference between any two array elements in spatially separated electromagnetic vector sensor array (SS-EVSA). Theoretical formulas describing the phase difference between array elements are derived from the phase descriptor and the geometric descriptor. Based on the characteristics of the phase difference, a new half-interval search MUSIC (HIS-MUSIC) algorithm is proposed. By searching half of the four-dimensional space, a joint estimation of the direction of arrival and polarization of the incident signal is obtained, which can effectively reduce the computational complexity of the joint estimation of the four-dimensional space. Finally, the efficiency of the algorithm is demonstrated by simulation experiments.

The paper by Li et al. (2017) proposed a novel algorithm for estimating the motion parameters of air maneuvering target by means of reconstructing time samples and signal. In this method, the received data of multiple antennas are first spliced together to reconstruct time samples of a single antenna by compensating a proper phase. Next, an ideal signal whose time sample number is equal to the length of the reconstructed time samples is constructed. At last, the estimation results of initial velocity and acceleration of the air maneuvering target are obtained by applying the nonlinear least squares method to compare the similarity between the reconstructed time samples and signal. This algorithm can achieve accurate parameter estimation with limited pulses.

The paper by Anil Kumar et al. (2016) considered the problem of spectrum blind reconstruction (SBR) and DOA estimation of constituent sources of a disjoint multi-band signal (MBS) at sub-Nyquist sampling rates. A simple modification to the receiver architecture by introducing an additional delay channel at every sensor is devised. Estimation algorithms based on ESPRIT is then employed to estimate the carrier frequencies, while MUSIC algorithm is employed to estimate their corresponding DOAs. With these parameters, the MBS spectrum is then reconstructed. A two-dimensional iterative grid refinement algorithm is also described to further improve the estimation accuracy. Identifiability issues are addressed and the conditions for unique identifiability are discussed. Numerical simulations are presented to show the validity of the proposed approach and compare the performance against appropriate bounds.

The paper by Amanat et al. (2017) addressed the problem of two-dimensional autoregressive estimation in the presence of additive white noise. The estimation method is developed by combining the low-order and high-order Yule-Walker (YW) equations. The noise-compensated YW equations are solved using an iterative algorithm. The proposed method is also applied to joint frequency and direction of arrival estimation in uniform linear arrays. It is shown by simulations that the performance of the proposed algorithm is evaluated and compared with other methods. 


\section{Beamforming for sensor arrays}

In this group, we have 2 papers. The paper by Wang et al. (2016) presented an enhanced eigenspace-based beamformer (ESB) by using the minimum sensitivity criterion. The proposed beamformer has significantly improved robustness against steering vector errors. The sensitivity function is defined as the squared norm of the appropriately scaled weight vector and since the sensitivity function of an array to perturbations becomes very large in the presence of steering vector errors, it can be used to find the best projection for the ESB, irrespective of the distribution of additive noises. As demonstrated by simulation results, the proposed beamformer has a better performance than the classic ESBs and the previously proposed uncertainty set based approach.

The paper by Ariyarathna et al. (2016) explored methods for synthesizing approximately frequency independent array factors at lower hardware complexity for wideband beamforming applications. The proposed approach employs 2-D infinite impulse response (IIR) digital beam filters together with nested uniform linear arrays (ULAs). The array is designed to have multiple levels of nesting. Each level of nesting consists of a ULA covering a temporal subband of the incident wideband signal. The use of nested arrays provides the required aperture size using a smaller number of elements compared to using a single ULA to capture the entire wideband signal. The use of different levels of nesting allows the operation of the digital processor for each sub-band at different clock rates. This is a hierarchical approach that saves both digital VLSI hardware and power consumption. The 2-D IIR digital beam filters that process each subband signal from each of the nested subarray achieves wideband beamforming. Simulations illustrate approximately frequency independent passbands as required in wideband beamforming.

\section{Signal processing in phased-array radar and MIMO radar}

In this group, we have 5 papers. The paper by Li et al. (2016b) proposed a novel wind speed estimation algorithm with airborne phased array weather radar for low altitude wind-shear, by combining space time adaptive processing and compressive sensing (CS). Thanks to the use of compressive sensing, the proposed method is able to estimate the wind speed accurately even with limited number of sampling pulses. The performance of the proposed algorithm is verified with numerical simulations.

In the paper by Liu and Wang (2016), a reweighted $L_{1}$ norm penalty algorithm for MMV problem in monostatic MIMO radar is proposed. The SVD technique is utilized to reduce the computational complexity and the sensitivity to noise. Then, a constrained minimization problem is formulated, and the coefficients of the RD-Capon spatial spectrum are exploited to design a weight matrix for reweighting $L_{1}$ norm penalty minimization. Finally, the DOAs can be obtained by solving the reweighted $L_{1}$ norm constraint minimization. Simulation results illustrate that this algorithm can provide better angle estimation performance than RD-Capon and $L_{1}$-SRACV algorithms.

In the paper by $\mathrm{Hu}$ et al. (2017), high resolution three-dimensional (3D) imaging method using MIMO radar with sparse array is studied. A method based on CS is firstly given. However, the CS-based method has the off-grid problem which will reduce the estimation accuracy of scatterers' position. Moreover, a high dimensional measurement matrix is required in the CS-based method, which will lead to a heavy storage and computation burden. To this end, a new method based on matrix completion is proposed. After reshaping the sparse $3 \mathrm{D}$ echo 
into a low-rank structured matrix, the full 3D echo can be recovered by solving a nuclear norm minimization problem. Then the accurate position of scatterers can be estimated by applying multi-dimensional harmonic retrieval methods to the full 3D echo. Finally, the high resolution $3 \mathrm{D}$ image of targets is reconstructed. The effectiveness of the method is validated by the results of comparative simulations.

In the paper by Shahbazi et al. (2017), a measurement matrix for CS-MIMO radar in the presence of clutter and interference is designed. To optimize the measurement matrix, three main criteria are considered simultaneously to improve detection and sparse recovery performance while suppressing clutter and interference. Due to the use of simultaneous multiobjective functions, a multi-objective optimization (MOO) framework is exploited. Some numerical examples are provided to illustrate the achieved improvement of our proposed method in target detection and sparse recovery performance. It is shown that the proposed MOO technique for measurement matrix design can achieve superior performance in target detection compared with Gaussian random measurement matrix technique.

In the paper by Fang et al. (2016), two nested algorithms, nested-ESPRIT and nested-ML, for joint DOD and DOA estimation in bistatic MIMO radar are proposed. The essence of the nested algorithms is to amalgamate signal grouping schemes with DOD/DOA estimation so that DODs and DOAs are iteratively and precisely estimated using only 1-D ESPRIT or 1-D MLE. Additionally, the estimated DODs and DOAs are automatically paired together without any overhead. Simulation results illustrate the superiority of the new algorithms in terms of the tradeoff between estimation accuracy and complexity.

Finally, we would like to thank all the authors and reviewers whose contributions have made this special issue possible. We would like to thank Prof. Zhiping Lin, past Editorin-Chief, Prof. Anton Kummert (editor) and Prof. Eric Rogers, present Editor-in-Chief, for approving the proposal of this Special Issue and for their support and guidance throughout the process. Warmest thanks also to Divya Devarajan and Susan Evans from Springer Journals Editorial Office for their tremendous work and support throughout the whole process of this Special Issue.

\section{References}

Ali Khan, N., Ali, S., \& Jansson, M. (2016). Direction of arrival estimation using adaptive directional time-frequency distributions. Multidimensional Systems and Signal Processing. https://doi.org/10.1007/ s11045-016-0435-y.

Amanat, A., Mahmoudi, A., \& Hatam, M. (2017). Two-dimensional noisy autoregressive estimation with application to joint frequency and direction of arrival estimation. Multidimensional Systems and Signal Processing. https://doi.org/10.1007/s11045-017-0539-z.

Anil Kumar, A., Razul, S. G., \& See, C.-M. (2016). Spectrum blind reconstruction and direction of arrival estimation of multi-band signals at sub-Nyquist sampling rates. Multidimensional Systems and Signal Processing. https://doi.org/10.1007/s11045-016-0455-7.

Ariyarathna, V., Madanayake, A., Agathoklis, P., \& Bruton, L. T. (2016). Mixed microwave-digital and multirate approach for wideband beamforming applications using 2-D IIR beam filters and nested uniform linear arrays. Multidimensional Systems and Signal Processing. https://doi.org/10.1007/s11045-0160422-3.

Astapov, S., Berdnikova, J., Ehala, J., Kaugerand, J., \& Preden, J.-S. (2017). Gunshot acoustic event identification and shooter localization in a WSN of asynchronous multichannel acoustic ground sensors. Multidimensional Systems and Signal Processing. https://doi.org/10.1007/s11045-017-0479-7.

Fang, W.-H., Tu, C.-C., \& Chen, Y.-T. (2016). Nested algorithms for joint DOD and DOA estimation in bistatic MIMO radar. Multidimensional Systems and Signal Processing. https://doi.org/10.1007/s11045017-0511-y.

Hu, X., Tong, N., He, X., \& Wang, Y. (2017). High resolution 3D imaging in MIMO radar with sparse array. Multidimensional Systems and Signal Processing. https://doi.org/10.1007/s11045-017-0531-7. 
Li, J., Lin, Z., Razul, S. G., Anil Kumar, A., Zheng, Y., \& See, C.-M. (2016a). DOA estimation with a rotational uniform linear array (RULA) and unknown spatial noise covariance. Multidimensional Systems and Signal Processing. https://doi.org/10.1007/s11045-016-0425-0.

Li, H., Zhou, M., Guo, Q., Wu, R., \& Xi, J. (2016b). Compressive sensing-based wind speed estimation for lowaltitude wind-shear with airborne phased array radar. Multidimensional Systems and Signal Processing. https://doi.org/10.1007/s11045-016-0448-6.

Li, H., Zhou, M., Wu, R., Zhang, Z., \& Zheng, J. (2017). Parameter estimation of air maneuvering target for multi-antenna system via reconstructing time samples and signal. Multidimensional Systems and Signal Processing. https://doi.org/10.1007/s11045-017-0495-7.

Liu, Q., \& Wang, X. (2016). Direction of arrival estimation via reweighted $l_{1}$ norm penalty algorithm for monostatic MIMO radar. Multidimensional Systems and Signal Processing. https://doi.org/10.1007/s11045016-0392-5.

Liu, S., Ynag, L., Li, D., Cao, H., \& Jiang, Q. (2016). 2D DOA estimation for noncircular sources using Lshaped sparse array. Multidimensional Systems and Signal Processing. https://doi.org/10.1007/s11045016-0402-7.

Romanofsky, R. R., \& Toonen, R. C. (2016). Past, present and future of ferroelectric and multiferroic thin films for array antennas. Multidimensional Systems and Signal Processing. https://doi.org/10.1007/s11045016-0449-5.

Shahbazi, N., Abbasfar, A., \& Jabbarian-Jahromi, M. (2017). Measurement matrix design for CS-MIMO radar using multi-objective optimization. Multidimensional Systems and Signal Processing. https://doi.org/10. 1007/s11045-017-0542-4.

Wang, J., Zhang, W., \& Liu, W. (2016). Minimum sensitivity based robust beamforming with eigenspace decomposition. Multidimensional Systems and Signal Processing. https://doi.org/10.1007/s11045-0160424-1.

Wu, N., Qu, Z., Si, W., \& Jiao, S. (2016). Joint estimation of DOA and polarization based on phase difference analysis of electromagnetic vector sensor array. Multidimensional Systems and Signal Processing. https: //doi.org/10.1007/s11045-016-0434-Z.

Zhang, X., He, Z., Liao, B., Zhang, X., \& Xie, J. (2017). DOA and phase error estimation using one calibrated sensor in ULA. Multidimensional Systems and Signal Processing. https://doi.org/10.1007/s11045-0170484-x.

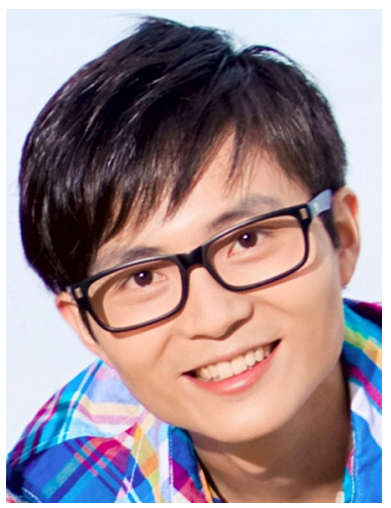

Bin Liao received the B.Eng. and M.Eng degrees from Xidian University, Xian, China, and the Ph.D. degree from The University of Hong Kong, Hong Kong, in 2006, 2009, and 2013, respectively, all in Electronic Engineering. From September 2013 to January 2014, he was a Research Assistant in the Department of Electrical and Electronic Engineering, The University of Hong Kong. From August 2016 to October 2016, he was a Research Scientist in the Department of Electrical and Electronic Engineering, The University of Hong Kong. He is currently an Associate Professor in the College of Information Engineering, Shenzhen University, Shenzhen, China. His research interests include sensor array processing, adaptive filtering, convex optimization, with applications to radar, navigation, and communications. He received the 2016 IEEE DSP Best Paper Award and 2017 IEEE DSP Best Paper Award. He is an Associate Editor of IEEE Transactions on Aerospace and Electronic Systems, IET Signal Processing, IEEE ACCESS, and Multidimensional Systems and Signal Processing. 


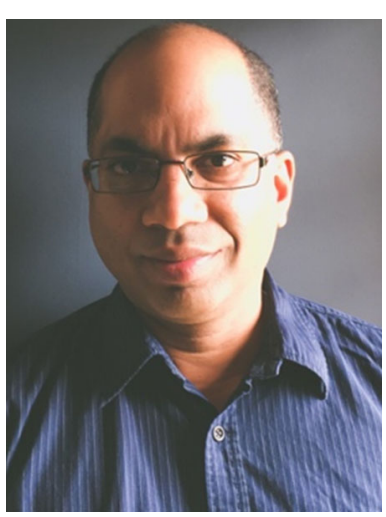

Arjuna Madanayake received the B.Sc. (First Class Hons.) degree in electronic and telecommunication engineering from the University of Moratuwa, Moratuwa, Sri Lanka, in 2002, and the M.Sc. and Ph.D. degrees in electrical engineering from the University of Calgary, Calgary, AB, Canada, in 2004 and 2008, respectively. He is currently a Associate Professor with the Department of Electrical and Computer Engineering, University of Akron, Akron, OH, USA. His current research interests include multidimensional signal processing, array processing for microwave and mm-wave systems, analog/digital and mixed-signal electronics, field-programmable gate array systems, and very large-scale integration for fast algorithms.

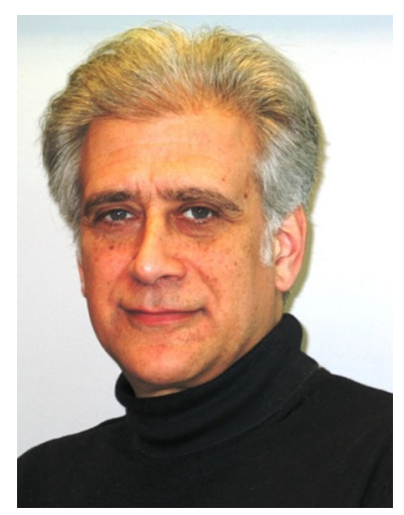

Panajotis Agathoklis received the Dipl.Ing. degree in electrical engineering and the Dr.Sc.Tech. degree from the Swiss Federal Institute of Technology, Zurich, Switzerland, in 1975 and 1980, respectively. From 1981 until 1983, he was with the University of Calgary as a PostDoctoral Fellow and part-time Instructor. Since 1983, he has been with the Department of Electrical and Computer Engineering, University of Victoria, B.C., Canada, where he is currently a Professor. His fields of interest are in control, digital signal processing and their applications. He worked in the stability of multidimensional systems and in the applications of 2D and 3D filtering. 